\title{
Avaliação de elementos inorgânicos em água e uma população humana exposta a rejeitos de minério
}

\author{
Inorganic element assessments in water and a human population \\ exposed to ore tailings
}

\author{
Francy Mendes Nogueira \\ Cardoso',ll* (iD)
}

Caio Rodrigues Telles ${ }^{1, \| 1}$

Wentony Mark Costa Milhomem" iD

Silas Vieira Trindade Junior"I (iD)

Luany Janaira Gois Vidal"

Roberto Messias Bezerral" (iD

Francinaldo Sarges Braga"l (iD

Huann Carllo Gentil

Vasconcelos" iD

Amanda Furtado de Almeida" iD

Silvia Eline Alfaia Lima" iD

Madson Ralide Fonseca

Gomes',"I iD

\section{RESUMO}

Introdução: A exploração do manganês no Amapá e o armazenamento inadequado dos rejeitos de minério na área portuária de Santana geraram impactos ambientais e sociais aos moradores locais. Objetivo: Avaliar a presença de elementos inorgânicos nas moradoras do bairro Elesbão expostas aos rejeitos de minérios. Método: Por meio da Espectrofotometria de Absorção Atômica de Chama (F-AAS), quantificaram-se metais na água utilizada pela população e na matriz biológica urina, enquanto no cabelo utilizou-se Espectrometria de Massas com Plasma Indutivamente Acoplado (ICP-MS). Coletou-se o sangue de 67 mulheres expostas e de 99 não expostas, destas, coletou-se 65 amostras de urina e 18 de cabelo. Foram realizadas análises hematológica, bioquímica e de coagulação no sangue de todas as participantes que também responderam a um questionário socioeconômico. Resultados: Quanto à faixa etária, 60,0\% das moradoras expostas estavam entre 18 e 42 anos e moravam na localidade entre 10 e 34 anos. Na água analisada, encontrou-se concentrações elevadas dos metais $\mathrm{As}, \mathrm{Cd}, \mathrm{Cu}, \mathrm{Fe}, \mathrm{Mn}$ e $\mathrm{Pb}$. Na matriz urina, observou-se significância para o Mn e, no cabelo, para $\mathrm{Pb}$ e $\mathrm{Mn}$. Dentre os parâmetros hematológicos significativos estavam Hb, Ht, VCM, HCM, monócitos, alterações morfológicas nas hemácias e plaquetas. Na avaliação bioquímica, observaram-se alterações nas enzimas fosfatase alcalina, bilirrubinas e creatinina. Conclusões: Há contaminação ambiental por elementos que podem comprometer a saúde da população exposta, quando em contato por tempo prolongado. Estudos mais aprofundados e direcionados à saúde da mulher precisam ser realizados, incluindo parâmetros como a análise de hormônios e marcadores genéticos, visando contribuir, assim, para uma ação das autoridades competentes em melhorar a saúde da população exposta.

PALAVRAS-CHAVE: Elementos Inorgânicos; Análise Laboratorial; Rio Amazonas; Elesbão; Amapá

\section{ABSTRACT}

Introduction: Manganese exploration in Amapá and inadequate ore tailing storage in the Santana port area have generated environmental and social impacts for residents. Objective: Assess the presence of inorganic elements in Elesbão neighborhood inhabitants exposed to ore tailings. Methods: Metals were quantified in the water used by the population and in urine by Flame Atomic Absorption Spectrophotometry (F-AAS), and in hair Inductively Coupled Plasma Mass Spectrometry (ICP-MS). Blood was obtained from 67 exposed and 99 unexposed women, totaling 65 urine and 18 hair samples. Hematological, biochemical and blood clotting analyses were performed in all participants, who also answered a socio-economic questionnaire. Results: Regarding age group, $60.0 \%$ of exposed residents ranged between 18 and 42 years old and lived in the area from 10 to 34 years. High concentrations of $\mathrm{As}, \mathrm{Cd}, \mathrm{Cu}, \mathrm{Fe}, \mathrm{Mn}$ and $\mathrm{Pb}$ were detected in the analyzed water. In urine, significance was observed for $\mathrm{Mn}$ and in hair, for $\mathrm{Pb}$ and $\mathrm{Mn}$. $\mathrm{Hb}, \mathrm{Ht}, \mathrm{VCM}, \mathrm{HCM}$, monocytes, morphological changes in red cells and platelets were among the significant hematological parameters. Regarding the biochemical evaluations, alterations in the enzymes Alkaline Phosphatase, Bilirubins and Creatinine were observed. Conclusions: 
Environmental contamination by elements that can compromise the health of the exposed population was observed. However, more in-depth studies aimed at women's health are required, including hormones and genetic marker analyses, thus contributing to the action of competent authorities to improve the health of the exposed population.

KEYWORDS: Elements; Laboratory Evaluation; Amazon River; Elesbão; Amapá

\section{INTRODUÇÃOO}

Muitos fatores sociais, econômicos e culturais existentes nas regiões do Brasil podem influenciar na determinação de vulnerabilidades da população, potencializando, assim, os efeitos da poluição ambiental, que causam riscos à saúde ${ }^{1}$.

Na Região Amazônica, foram implantados empreendimentos produtivos através de planos e políticas de desenvolvimento do Governo Federal, como a Indústria e Comércio de Minérios S/A (Icomi), que explorava o minério de manganês na Serra do Navio, no estado do Amapáz. Este minério era processado e escoado na área industrial e portuária no município de Santana, onde funcionava a usina de pelotização. Os rejeitos oriundos desta atividade foram depositados inadequadamente no local, permanecendo a céu aberto até os dias atuais. Representa, assim, relevante risco de exposição à população residente no entorno da área da empresa, inclusive o bairro do Elesbão ${ }^{3,4}$.

O conhecimento dos efeitos nocivos das interações dos elementos inorgânicos com a saúde humana é imprescindível, pois provocam impactos físicos, biológicos e socioeconômicos e geram danos à saúde, uma vez que são elementos amplamente encontrados no solo, na água, no ar, no lixo, nos alimentos e nos sedimentos. A intervenção humana por meio de atividades industriais é a principal responsável pelo lançamento de metais à natureza, contaminando as águas, as plantas e os animais, pois os metais possuem níveis elevados de reatividade e bioacumulação ${ }^{5,6,7}$. Cada elemento inorgânico possui uma característica e uma especificidade por órgãos e tecidos, porém geralmente tem predileção pelos tecidos hepático e renal, onde ocorrem os processos de desintoxicação celular, como também pelo sistema reprodutivo ${ }^{8}$.

De acordo com os indicadores epidemiológicos brasileiros, as mulheres adoecem mais frequentemente do que os homens e são mais predispostas à intoxicação9,10. Um estudo realizado no estado da Bahia demonstrou alterações citogenéticas em mulheres expostas à poluição ambiental pela presença dos elementos como chumbo e cádmio ${ }^{11}$. Além disso, estudos demonstraram que as diferenças dos mecanismos epigenéticos entre os sexos influenciam na desintoxicação e biotransformação de substâncias tóxicas ${ }^{12}$.

Nesse contexto, o presente trabalho visa avaliar a presença dos elementos inorgânicos no ambiente e nas mulheres do bairro do Elesbão. Para tal, foram utilizados biomarcadores hematológicos e bioquímicos nos indivíduos expostos, bem como a avaliação da presença de manganês $(\mathrm{Mn})$, chumbo $(\mathrm{Pb})$ e outros elementos tóxicos nas matrizes biológicas urina e cabelo, as quais são importantes no monitoramento de sistema de advertência às doenças, almejando a prevenção de problemas de saúde ${ }^{13,14}$.

\section{MÉTODO}

Área e tipo de estudo

Realizado com mulheres voluntárias residentes no bairro Elesbão há pelo menos 10 anos, localizado próximo a uma mineradora do município de Santana, estado do Amapá. As do grupo não exposto aos metais residiam em Macapá e todas estavam entre 18 e 65 anos de idade. Conforme o Instituto Brasileiro de Geografia e Estatística, em 2010, a população do Elesbão era de 4.737 habitantes, sendo 2.332, da população feminina. Este estudo é transversal quantitativo, permitindo avaliação epidemiológica e laboratorial das moradoras do Elesbão expostas ao rejeito de minério.

\section{Amostragem}

Após assinarem o Termo de Consentimento Livre e Esclarecido, com as normas da Resolução n 196, de 10 de outubro de 1996 do Conselho Nacional de Saúde (CNS), as participantes do estudo responderam a um questionário socioeconômico e coletaram sangue, cabelo e urina.

Foram coletadas 283 amostras de sangue, sendo 109 de mulheres expostas e 174 de não expostas e, após passarem pelos critérios de exclusão, ficaram 67 amostras pertencentes a mulheres expostas e 99 amostras de não expostas, aptas ao estudo. Não participaram da pesquisa as mulheres fumantes, mulheres que tinham realizado procedimentos químicos no cabelo, trabalhadoras de fábrica de baterias, de fundição, de polimento e refinamento de metais, soldagem, dentre outros. Informações estas retiradas do questionário socioeconômico que serviram como critério de exclusão, uma vez que se procurou selecionar moradoras que sabidamente não realizavam atividades relacionadas com o manuseio de metais.

A aplicação dos questionários e as coletas foram realizadas na Unidade Básica de Saúde do bairro Elesbão e no Laboratório de Análises Clínicas II da Universidade Federal do Amapá (Unifap), no período de novembro de 2018 a junho de 2019. Em todas as amostras de sangue, foram realizados exames laboratoriais para análise hematológica, bioquímica e de coagulação. Realizou-se dosagem dos elementos $\mathrm{Pb}, \mathrm{Mn}$ e cobre ( $\mathrm{Cu}$ ) em 65 amostras de urina através da Espectrofotometria de Absorção Atômica com Chama (FAAS) no Laboratório de Absorção Atômica e Bioprospecção (LAAB) da Unifap e de $\mathrm{Pb}, \mathrm{Mn}$, $\mathrm{Cu}$, ferro ( $\mathrm{Fe}$ ), cádmio (Cd) e arsênio (As) em 18 amostras de cabelo por Espectrometria de Massas com Plasma Indutivamente Acoplado (ICP-MS) no Laboratório de Espectrometria Atômica (LABSPECTRO) do Departamento de Química da Pontifícia Universidade Católica do Rio de Janeiro (PUC-RJ). 


\section{Coleta da água}

As amostras de água foram coletadas no período chuvoso. Foi coletado um litro de água em dez pontos diferentes do bairro, incluindo igarapés, em recipientes plásticos apropriados, devidamente identificados. As amostras foram transportadas em caixas térmicas com gelo seco até o laboratório, onde permaneceram sob refrigeração $\left(4^{\circ} \mathrm{C}-8^{\circ} \mathrm{C}\right)$ até a análise. 0 preparo das amostras seguiu os padrões estabelecidos pela American Public Health Association (APHA) ${ }^{15}$.

Após homogeneização e filtração, as amostras foram colocadas no espectrofotômetro de FAAS, modelo AA-6300 para determinação dos metais Cd, As, Cu, Fe, zinco (Zn), Pb e Mn no LAAB da Unifap ${ }^{16,17}$.

Foram determinadas curvas de calibração para cada metal analisado a partir de soluções padrão certificadas para FAAS fornecidas pelo Instituto Nacional para Ciência e Tecnologia dos Estados Unidos da América (NIST-USA). As análises foram realizadas em triplicata e o resultado expresso em $\mathrm{mg} \cdot \mathrm{L}^{-1}$. As concentrações máximas permitidas (CMP) de metais em água doce estão estabelecidas na Resolução do Conselho Nacional do Meio Ambiente (Conama) $n^{\circ} 357$, de 17 de março de $2005^{18}$.

\section{Coleta do material biológico (sangue, cabelo e urina)}

Foi realizada coleta de sangue por meio de punção venosa a vácuo em tubos contendo anticoagulantes EDTA K3 para os exames de hemograma, citrato de sódio $3,2 \%$ para tempo de protrombina (TP) e tempo de tromboplastina parcial ativado (TTPA) e heparina, para determinação de metais pesados. Para as análises bioquímicas, os tubos não continham anticoagulante ${ }^{6,19}$.

A análise sanguínea foi realizada no Laboratório de Análises Clínicas II da Unifap. O hemograma foi realizado em analisador hematológico Icounter 3D e foram preparados esfregaços sanguíneos para avaliação diferencial e morfológica celular. Os exames de coagulação foram realizados em coagulômetro Clo Timer. As análises bioquímicas foram feitas no analisador automático LANDWIND LW C200i com kits de reagentes Labtest.

Coletou-se cerca de $500 \mathrm{mg}$ de cabelo da região occipital com auxílio de uma tesoura de titânio, a uma distância de até $2 \mathrm{~cm}$ do escalpo. As amostras foram acondicionadas em invólucros de polietileno fechados hermeticamente com zíper devidamente identificados, mantidos em local seco e limpo e transportados para o LABSPECTRO

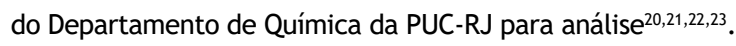

As amostras de urina foram coletadas pelas participantes da pesquisa em frascos próprios, devidamente identificados, armazenados e transportados. Permaneceram congeladas a $-80^{\circ} \mathrm{C}$ até a análise, realizada no LAAB/Unifap 6 .

\section{Preparo das amostras para análise}

As amostras de urina foram diluídas $1+7$ em ácido nítrico 0,2\% ( $\mathrm{v} / \mathrm{v})$, conforme metodologia utilizada por Marinha ${ }^{24}$. Após homogeneização, as medições foram realizadas em triplicata para cada metal no equipamento FAAS modelo AA-6300.
Cada amostra de cabelo (cerca de $200 \mathrm{mg}$ a $250 \mathrm{mg}$ ) foi submetida a lavagem, de acordo com metodologia adaptada da Agência Internacional de Energia Atômica (IAEA), na qual foram utilizadas água ultrapura e acetona. Primeiramente lavou-se uma vez com acetona, três vezes com a água ultrapura e mais uma vez com acetona. As amostras permaneceram submersas por $10 \mathrm{~min}$ em banho ultrassônico em cada solvente. Após a lavagem, as amostras secaram em estufa a $50^{\circ} \mathrm{C}$ por $12 \mathrm{~h}$.

As amostras foram pesadas após secagem e, de acordo com a massa remanescente, foram separadas em grupos e determinadas as quantidades de ácido nítrico a $65 \%$ e peróxido de hidrogênio que seriam utilizados na etapa de digestão.

Após a adição de ácido nítrico, as amostras foram secas em placas de aquecimento a $80^{\circ} \mathrm{C}$ por $12 \mathrm{~h}$. Em seguida, adicionou-se o peróxido de hidrogênio e foi realizada nova etapa de secagem por $20 \mathrm{~min}$ a $80^{\circ} \mathrm{C}$. Após atingirem a temperatura ambiente, as amostras foram diluídas com água ultrapura na mesma proporção utilizada para o peróxido. Para finalmente serem analisadas no equipamento de ICP-MS do LABSPECTRO do Departamento de Química da PUC-RJ.

\section{Considerações éticas}

O estudo foi aprovado pelo Comitê de Ética e Pesquisa da Unifap, por meio do parecer $n^{\circ} 2853167$ e $n^{\circ}$ CAE 94256218.0.0000.0003, em 18 de julho de 2018.

Tratamento dos dados e análises

Foram realizadas média e desvio-padrão pelo Microsoft Excel 2010. Pelo programa BioEstat 5.0, foi realizado o teste de normalidade Kolmogorov-Smirnov, em seguida, para a análise dos dados paramétricos, foi realizado o teste $t$-student ( $\mathrm{t}$ ) e para os não paramétricos, o Mann-Whitney (U). 0 nível de significância considerado foi de $p=0,05$.

\section{RESULTADOS E DISCUSSÃO}

\section{Questionário socioeconômico}

Neste estudo observou-se que $40,9 \%$ das mulheres residiam no Elesbão entre 22 e 34 anos. Dentre as características socioeconômicas, destaca-se que $60,0 \%$ das mulheres pertenciam a faixa etária entre 18 e 42 anos, em ambos os grupos. Quanto à escolaridade, cerca de $40,0 \%$ das mulheres expostas concluíram o ensino médio, seguido de $28,0 \%$ com ensino fundamental incompleto, enquanto $30,3 \%$ das não expostas apresentaram ensino superior incompleto, seguido de $26,2 \%$ com ensino médio completo. Das que trabalhavam, a maioria possuía renda mensal de até meio salário-mínimo no grupo exposto, de até um salário mínimo no não exposto e a maioria não tinha renda mensal.

Estudos mostraram que o nível de escolaridade e renda são fatores que impactam diretamente na percepção de saúde das pessoas, afetando de forma negativa o estado de saúde dos indivíduos que moram em regiões com muita desigualdade, reduzindo em torno 
de $4 \%$ a chance de serem saudáveis quando comparados com os que moram em regiões menos desiguais ${ }^{25}$. Indivíduos com níveis mais elevados de escolaridade e renda têm mais acesso a bens e serviços de saúde, o que thes proporciona melhor qualidade de vida ${ }^{26}$.

Aágua de consumo mais utilizada em ambos os grupos foi a de poço, seguida pela da rede de abastecimento e apenas quatro mulheres expostas utilizavam a água do rio como única fonte de consumo.

Entre os sintomas relatados pelas mulheres expostas estão: anemia, dores musculares, instabilidade emocional e distúrbios do sono e do trato gastrointestinal (TGI). Em relação à fertilidade, 25,4\% das mulheres relataram ter diminuição da libido e 20,0\% já tiveram aborto. Entre os casais, 16,0\% foram considerados estéreis. Quanto à incidência de câncer em pessoas da família, $48,0 \%$ das mulheres responderam positivamente, sendo que $30,0 \%$ eram de colo do útero.

Os metais pesados $(\mathrm{As}, \mathrm{Cd}, \mathrm{Pb})$ causam efeitos sobre a infertilidade humana e malformações congênitas, havendo interferência no desenvolvimento neurológico do embrião, abortos, partos prematuros e alterações hormonais ${ }^{27}$.

\section{Análise da água}

Observaram-se alterações em vários pontos de coleta (Tabela 1). Os metais $\mathrm{As}, \mathrm{Cu}, \mathrm{Fe}, \mathrm{Mn}, \mathrm{Cd}$ e Pb exibiram concentrações acima dos valores máximos permitidos pela legislação vigente. Fato preocupante, pois são potencialmente tóxicos, arriscando a saúde da população que utiliza a água.

Estudos mostraram que há contaminação por metais pesados no Igarapé que percorre o bairro do Elesbão e que deságua no Rio Amazonas, no qual foram encontradas concentrações elevadas de As e $\mathrm{Fe}^{2}$, corroborando com os resultados deste estudo.

Conforme outros estudos de análise da água realizados no bairro do Elesbão, no período do verão amazônico, foram demonstrados elevados índices de $\mathrm{Mn}$ e $\mathrm{Fe}^{13,14}$. O verão amazônico é um período de estiagem em que ocorre pouca ou nenhuma chuva, enquanto o inverno amazônico é um período chuvoso, no qual há elevação das águas dos rios possibilitando o desprendimento das concentrações de metais que possivelmente se encontravam no sedimento. No presente estudo, realizado durante o inverno amazônico, encontramos concentrações de diferentes metais.

Em estudo de Facundes ${ }^{4}$, foi relatado alto teor de As em dois poços localizados no fundo da barragem de rejeitos. Contudo, os resultados de amostragens efetuadas de outros pontos de coleta de água para o consumo revelaram que os teores de As foram maiores no Rio Amazonas do que nos poços abertos por particulares no entorno da área industrial da Icomi. Esses valores de As variaram bastante ao longo do ano, sendo maiores nas estações chuvosas, indicando que o Rio Amazonas era um importante transportador de $\mathrm{As}^{4}$.

Análise dos metais nas matrizes biológicas (urina e cabelo)

Nas concentrações médias achadas nesta pesquisa (Tabela 2), observou-se diferença significativa entre os grupos para o Mn urinário $(\mathrm{p}<0,0001)$ e para o $\mathrm{Pb}$ e o Mn no cabelo $(\mathrm{p}<0,05)$.

Quanto à concentração média do $\mathrm{Pb}$ urinário, observou-se que o valor médio encontrado no grupo de mulheres expostas está dentro do valor de referência e no grupo de mulheres não expostas, esse metal não foi detectado.

O Pb é um metal tóxico que se acumula no organismo e pode causar danos ao sistema nervoso central, alterando as funções neurocognitivas e neurofisiológicas tanto em crianças quanto em adultos. Estudos demonstraram que não há nível seguro da presença do $\mathrm{Pb}$ sem prejuízo ao organismo, tornando-se uma preocupação para saúde pública qualquer fonte de exposição a este metal ${ }^{29}$.

$\mathrm{O}$ organismo exposto ao $\mathrm{Pb}$ por longo período tem como resultado a inibição da síntese da hemoglobina, além da redução do tempo de circulação das hemácias, o que causa anemia leve a moderada em adultos e severa em crianças $^{30}$. Anemia foi uma das patologias relatadas pelas mulheres, sendo que cerca de $70,0 \%$ estavam no

Tabela 1. Resultados da média e desvio-padrão das concentrações dos metais em mg. L $^{-1}$ analisados nos dez pontos de coleta do bairro Elesbão, no período chuvoso da região.

\begin{tabular}{|c|c|c|c|c|c|c|c|}
\hline \multirow{2}{*}{ Pontos de Coleta } & $\mathrm{Cu}\left(\mathrm{mg} \cdot \mathrm{L}^{-1}\right)$ & $M n\left(m g . L^{-1}\right)$ & $\mathrm{Fe}\left(\mathrm{mg} \cdot \mathrm{L}^{-1}\right)$ & $\mathrm{Cd}\left(\mathrm{mg} \cdot \mathrm{L}^{-1}\right)$ & $\mathrm{Pb}\left(\mathrm{mg} \cdot \mathrm{L}^{-1}\right)$ & As $\left(\mathrm{mg} \cdot \mathrm{L}^{-1}\right)$ & Zn (mg. $\left.\mathrm{L}^{-1}\right)$ \\
\hline & $(M D \pm D P)$ & $(M D \pm D P)$ & $(M D \pm D P)$ & $(M D \pm D P)$ & $(M D \pm D P)$ & $(M D \pm D P)$ & $(M D \pm D P)$ \\
\hline VR Conama* & 0,009 & 0,1 & 0,3 & 0,001 & 0,01 & 0,01 & 0,18 \\
\hline Ponto 1 & $0,033 \pm 0,018$ & $0,046 \pm 0,005$ & $0,905 \pm 0,003$ & $0,002 \pm 0,001$ & $0,058 \pm 0,006$ & $1,4 \pm 0,1$ & $0,053 \pm 0,001$ \\
\hline Ponto 2 & $0,048 \pm 0,017$ & $0,071 \pm 0,001$ & $0,295 \pm 0,004$ & ND & ND & $2,6 \pm 1,2$ & $0,032 \pm 0,002$ \\
\hline Ponto 3 & $0,014 \pm 0,014$ & $0,061 \pm 0,003$ & $0,916 \pm 0,029$ & ND & ND & $3,8 \pm 0,9$ & $0,029 \pm 0,002$ \\
\hline Ponto 4 & $0,022 \pm 0,012$ & $0,110 \pm 0,010$ & $0,910 \pm 0,011$ & ND & ND & $5,4 \pm 0,9$ & $0,035 \pm 0,003$ \\
\hline Ponto 5 & $0,017 \pm 0,011$ & $0,041 \pm 0,003$ & $0,489 \pm 0,001$ & ND & ND & $7,5 \pm 0,7$ & $0,007 \pm 0,003$ \\
\hline Ponto 6 & $0,025 \pm 0,010$ & $0,049 \pm 0,002$ & $0,821 \pm 0,052$ & ND & ND & $10,9 \pm 0,9$ & $0,01 \pm 0,002$ \\
\hline Ponto 7 & $0,012 \pm 0,01$ & $0,014 \pm 0,003$ & $0,034 \pm 0,005$ & ND & ND & $10,3 \pm 1,0$ & $0,026 \pm 0,001$ \\
\hline Ponto 8 & $0,019 \pm 0,009$ & $0,027 \pm 0,007$ & $0,069 \pm 0,006$ & ND & ND & $11,4 \pm 1,1$ & $0,018 \pm 0,004$ \\
\hline Ponto 9 & $0,025 \pm 0,009$ & $0,344 \pm 0,016$ & $1.666 \pm 0,104$ & ND & ND & $13,9 \pm 0,9$ & $0,018 \pm 0,005$ \\
\hline Ponto 10 & $0,024 \pm 0,009$ & $0,055 \pm 0,001$ & $0,846 \pm 0,055$ & ND & ND & $16,9 \pm 1,2$ & $0,008 \pm 0,002$ \\
\hline
\end{tabular}

Fonte: Elaborada pelos autores, 2021.

* Valores de referência em mg. $\mathrm{L}^{-1}$ segundo Resolução Conama n ${ }^{\circ}$ 357, de 17 de março de $2005^{18}$.

ND: não detectado; $M D \pm \mathrm{DP}$ : média e desvio-padrão. 
Tabela 2. Média das concentrações de $\mathrm{Pb}, \mathrm{Cu}, \mathrm{Mn}, \mathrm{Cd}$, As e Fe em urina e cabelo nos grupos compostos por mulheres expostas ( $\mathrm{n}=29$ ) e não expostas $(n=36)$. Valores médios $(M D)$, desvio-padrão (DP), teste estatístico e valor de $p$.

\begin{tabular}{|c|c|c|c|c|c|}
\hline \multirow{3}{*}{ Metais } & \multirow{3}{*}{ VR } & \multicolumn{2}{|c|}{ Grupo } & \multirow{3}{*}{ Resultado do teste } & \multirow{3}{*}{ p-valor } \\
\hline & & Exposto & Não exposto & & \\
\hline & & $(M D \pm D P)$ & $(M D \pm D P)$ & & \\
\hline \multicolumn{6}{|l|}{ Urina } \\
\hline $\mathrm{Pb}$ & até $50,00 \mu \mathrm{g} \cdot \mathrm{g}^{-1}$ & $16,90 \pm 54,30$ & 0,00 & $U=473$ & 0,0800 \\
\hline $\mathrm{Cu}$ & até $60,00 \mu \mathrm{g} \cdot \mathrm{L}^{-1}$ & $45,40 \pm 84,20$ & $34,9 \pm 49,70$ & $U=512,5$ & 0,9000 \\
\hline $\mathrm{Mn}$ & até $10,00 \mu \mathrm{g} \cdot \mathrm{L}^{-1}$ & $29,00 \pm 33,40$ & $117,7 \pm 46,70$ & $t=-8,4$ & $<0,0001$ \\
\hline \multicolumn{6}{|l|}{ Cabelo } \\
\hline $\mathrm{Pb}$ & até $9,30 \mathrm{mg} \cdot \mathrm{L}^{-1}$ & $2,40 \pm 2,70$ & $0,3 \pm 0,30$ & $U=13,5$ & 0,0200 \\
\hline $\mathrm{Cd}$ & até $0,30 \mathrm{mg} \cdot \mathrm{L}^{-1}$ & $0,07 \pm 0,05$ & $0,01 \pm 001$ & $U=19,5$ & 0,0800 \\
\hline $\mathrm{Cu}$ & 10,00 a $32,00 \mathrm{mg} \cdot \mathrm{L}^{-1}$ & $11,90 \pm 4,30$ & $8,4 \pm 2,00$ & $U=18,0$ & 0,0600 \\
\hline $\mathrm{Mn}$ & 0,15 a $1,20 \mathrm{mg} \cdot \mathrm{L}^{-1}$ & $4,70 \pm 3,00$ & $0,6 \pm 0,70$ & $\mathrm{U}=7,0$ & 0,004 \\
\hline As & até $0,15 \mathrm{mg} \cdot \mathrm{L}^{-1}$ & $0,03 \pm 0,01$ & $0,02 \pm 0,01$ & $t=1,3$ & 0,1000 \\
\hline $\mathrm{Fe}$ & 7,00 a $18,00 \mathrm{mg} \cdot \mathrm{L}^{-1}$ & $34,90 \pm 17,40$ & $23,1 \pm 28,70$ & $t=0,9$ & 0,3000 \\
\hline
\end{tabular}

Fonte: Elaborada pelos autores, 2021.

VR: valor de referência; $M D \pm D P$ : média e desvio-padrão; U: Teste estatístico Mann-Whitney; t: Teste estatístico t-student.

VR da urina - VR para Pb segundo a Norma Regulamentadora $n^{\circ} 7^{59}$ e VR do Cu e Mn segundo Burtis e Burns ${ }^{28}$.

VR do cabelo - VR segundo dados obtidos do LABSEPCTRO do Departamento de Química da PUC-RJ.

grupo exposto e $35,0 \%$ no não exposto, porém não foi detectada a presença do metal na urina dessas últimas. Apesar de detectado apenas em um ponto da coleta de água, é um dado relevante que merece mais investigação, ressaltando que não deveria ser encontrado no organismo, como constatado no presente estudo.

Segundo Ramos ${ }^{6}$, ao avaliar exposição ambiental ao Mn em uma população residente próxima a um estaleiro em Angra dos Reis, RJ, as concentrações médias desse metal na urina não tiveram diferenças significativas com resultados dentro do intervalo de referência. Esses resultados não corroboram com o presente estudo.

Entretanto, em outro estudo que avaliou o nível de exposição a metais em trabalhadores da construção naval, observou-se que cerca de $50 \%$ das amostras de urina continham $\mathrm{Mn}$ acima do valor de referência, indicando a exposição ${ }^{31}$.

A contaminação via oral por $\mathrm{Mn}$ em excesso causa danos ao organismo. Alimentos ricos em Mn podem ser de ordem vegetal, como arroz, trigo, cereiais, além de frutos do mar e água. Condições encontradas não só no município de Santana, mas também em Macapá, banhada pelo Rio Amazonas. 0 sedimento analisado nos períodos chuvoso e de estiagem na orla de Macapá continha metais pesados em várias concentrações, como As, $\mathrm{Zn}, \mathrm{Ni}$ e $\mathrm{Mn}^{32}$.

As concentrações médias de $\mathrm{Mn}$ em cabelo de adultos residentes em área localizada nas proximidades de uma indústria de ligas de ferro-manganês, na Bahia, estavam cerca de oito vezes mais elevadas que o valor preconizado. Demonstrou-se que a medição do $\mathrm{Mn}$ no cabelo é um importante biomarcador ambiental ${ }^{33}$, corroborando com o presente estudo, no qual a média da concentração de $\mathrm{Mn}$ em cabelo das moradoras do Elesbão está cerca de quatro vezes acima do valor preconizado.

Outros estudos encontraram índices elevados de $\mathrm{Mn}$ em cabelos de crianças expostas a minérios em uma comunidade da Bahia, apresentando 15,20 $\mu \mathrm{g} \mathrm{Mn} / \mathrm{g}$ cabelo, enquanto a população não exposta apresentou 1,37 $\mu \mathrm{g} \mathrm{Mn} / \mathrm{g}$ cabelo $^{33,34}$. Evidenciando que quanto maior a exposição maior será o acúmulo do elemento no organismo.

A análise de $\mathrm{Pb}$ em cabelo das moradoras do Elesbão demonstrou significância entre os grupos estudados, sendo que o grupo exposto apresentou resultados cerca de seis vezes maior que o não exposto, embora ambos estejam dentro do intervalo de referência. Fator preocupante, pois se encontrou índice elevado de $\mathrm{Pb}$ em um ponto de coleta da água do rio, caracterizando exposição ambiental.

Após o rompimento da barragem de minérios em Mariana, evidenciou-se aumento das concentrações de metais como $\mathrm{Pb}$ e $\mathrm{Mn}$ nas amostras de cabelo da população local quando comparadas a períodos anteriores. Além disso, o consumo de água e alimentos provenientes do rio contaminado pode influenciar nesses índices ${ }^{35}$.

A análise em cabelo de moradores das comunidades das ithas da Volta Grande do Rio Xingu e de Belo Monte e Altamira no Pará destacou $\mathrm{Pb}$ e mercúrio em concentrações médias elevadas. Embora seja área de garimpo, ressaltamos a importância da contaminação da água do rio utilizada pela população, a qual apresentou maior concentração média de $\mathrm{Pb}$ com cerca de quatro vezes o valor médio dos outros tipos de água ${ }^{36}$.

Assim, destacamos que o bairro do Elesbão também está suscetível à contaminação como mostra o resultado nas amostras de cabelo desta população.

\section{Avaliação hematológica}

Foi encontrada diferença significativa $(p<0,05)$ entre os grupos estudados nos parâmetros: hemoglobina $(\mathrm{Hb})$, hematócrito $(\mathrm{Ht})$, volume globular médio (VCM) e hemoglobina corpuscular média ( $\mathrm{HCM}$ ), com indivíduos do grupo exposto apresentando médias ligeiramente inferiores às do grupo não exposto. Em relação aos monócitos $(p=0,0494)$, ambos os grupos estavam com valores 
médios ligeiramente inferiores aos valores de referência; e no tempo de protombina (TP), na razão de normatização internacional (INR) e no tempo de tromboplastina parcial ativada (TTPA), o valor médio está dentro dos valores preconizados. Além da concentração média do volume plaquetário médio (VPM), que se encontra discretamente acima do valor de referência (Tabela 3).

As alterações hematológicas evidenciam-se na intoxicação por metais como o $\mathrm{Pb}$, o As, o $\mathrm{Fe}$ e o Cd. Na intoxicação crônica por $\mathrm{Pb}$ encontramos uma anemia normocítica normocrômica ou microcítica e hipocrômica com síntese diminuída da heme e hemólise aumentada. Também ocorre anisocitose e poiquilocitose, hemácias nucleadas, além de policromasia ${ }^{37}$. Essas alterações descritas acima corroboram com os resultados encontrados neste trabalho.

Um estudo que observou 192 pacientes com história de envenenamento por $\mathrm{Pb}$ durante 50 anos, comparando-os com um grupo controle, demonstrou que os indivíduos expostos tinham sete vezes mais chance de desenvolver hipertensão e hematócrito mais baixo que os indivíduos do grupo controle ${ }^{38}$.

Correlacionando um estudo realizado na população exposta do Elesbão, encontrou-se diferença significativa no parâmetro hematócrito ${ }^{13,14}$. Porém, no presente estudo, os outros parâmetros hematimétricos que tiveram significância, não foram evidenciados no estudo citado ${ }^{14}$, podendo estar relacionado ao $\mathrm{n}$ amostral, que no presente estudo é maior.

Os monócitos são células mieloides que derivam da mesma célula progenitora dos neutrófilos. Após formação na medula óssea, são liberados na corrente sanguínea e migram para os tecidos, formando o sistema reticuloendotelial, importante no combate aos microorganismos ${ }^{39,40}$.

O estudo de Lima apresentou significância entre os grupos apenas quanto aos linfócitos com média ligeiramente inferior no grupo exposto ${ }^{13,14}$. Comparando com o presente estudo, foi observada significância na concentração dos monócitos, embora as concentrações médias dos neutrófilos, linfócitos e monócitos estejam dentro dos valores de referência e menores no grupo exposto, indicando leve alteração nas células de defesa do organismo da população estudada.

Conforme estudo que avaliou exposição crônica ao $\mathrm{Pb}$, não foi encontrada diferença significativa nos níveis de linfócitos e neutrófilos entre os grupos estudados, corroborando com o presente estudo ${ }^{41}$.

O plaquetograma deste estudo não apresentou significância como ocorrido em outro estudo envolvendo a mesma população ${ }^{13,14}$. Atribuiu-se essa discordância ao número amostral de cada estudo, já que neste foram analisados 67 indivíduos no

Tabela 3. Análise dos parâmetros hematológicos (hemograma e coagulograma) em grupo exposto $(n=67)$ e não exposto $(n=99)$ apresentando os valores médios $(M D)$, desvio-padrão (DP), teste estatístico e valor de p.

\begin{tabular}{|c|c|c|c|c|c|}
\hline \multirow{3}{*}{$\begin{array}{l}\text { Parâmetros } \\
\text { hematológicos }\end{array}$} & \multirow{3}{*}{$V^{*}$} & \multicolumn{2}{|c|}{ Grupo } & \multirow{3}{*}{ Resultado do teste } & \multirow{3}{*}{ p-valor } \\
\hline & & Exposto & Não exposto & & \\
\hline & & $M D \pm D P$ & $M D \pm D P$ & & \\
\hline Eritrócitos (x 1012/L) & 3,8 a 4,8 & $4,7 \pm 0,3$ & $4,8 \pm 0,3$ & $t=-0,4$ & 0,6860 \\
\hline Hemoglobina (g/dL) & 12,0 a 15,0 & $12,5 \pm 1,1$ & $13,0 \pm 0,9$ & $U=2.623,0$ & 0,0220 \\
\hline Hematócrito (\%) & 36,0 a 46,0 & $39,2 \pm 2,9$ & $40,4 \pm 2,3$ & $U=2.494,0$ & 0,006 \\
\hline VCM (fL) & 83,0 a 101,0 & $82,1 \pm 4,9$ & $84,1 \pm 4,3$ & $t=-2,7$ & 0,0070 \\
\hline $\mathrm{HCM}(\mathrm{pg})$ & 27,0 a 32,0 & $26,4 \pm 0,8$ & $27,0 \pm 1,5$ & $\mathrm{t}=-2,7$ & 0,0070 \\
\hline CHCM (g/dL) & 31,5 a 34,5 & $31,9 \pm 0,5$ & $31,8 \pm 2,9$ & $U=2.986,5$ & 0,2770 \\
\hline RDW CV (\%) & 11,0 a 14,0 & $10,8 \pm 0,8$ & $11,0 \pm 3,0$ & $U=2.986,5$ & 0,9750 \\
\hline Leucócitos (/mm³) & $3.500,0$ a $10.000,0$ & $5.482,0 \pm 1.177,0$ & $5.715,0 \pm 1.341,0$ & $t=-1,1$ & 0,2570 \\
\hline Neutrófilos $\left(/ \mathrm{mm}^{3}\right)$ & $1.700,0$ a $8.000,0$ & $3.105,0 \pm 921,0$ & $3.394,0 \pm 1.079,0$ & $t=-1,6$ & 0,0950 \\
\hline Linfócitos (/mm³) & 900,0 a $2.900,0$ & $1.841,0 \pm 464,0$ & $1.890,0 \pm 489,0$ & $t=-0,6$ & 0,5180 \\
\hline Monócitos (/mm³) & 300,0 a 900,0 & $241,0 \pm 219,0$ & $264,0 \pm 108,0$ & $U=2.719,0$ & 0,0400 \\
\hline Eosinófilos (/mm³) & 50,0 a 500,0 & $213,0 \pm 222,0$ & $174,0 \pm 121,0$ & $U=3.266,0$ & 0,8000 \\
\hline Basófilos (/mm³) & até 200,0 & $0,7 \pm 6,3$ & $0,5 \pm 5,5$ & $\mathrm{t}=0,2$ & 0,8000 \\
\hline Plaquetas $\left(/ \mathrm{mm}^{3}\right)$ & $140.000,0$ a $450.000,0$ & $248.700,0 \pm 58.900,0$ & $250.800,0 \pm 49.200,0$ & $t=-0,2$ & 0,8000 \\
\hline $\operatorname{VPM}\left(\mu \mathrm{m}^{3}\right)$ & 7,4 a 10,4 & $10,5 \pm 0,6$ & $10,4 \pm 0,9$ & $U=2.991,5$ & 0,2000 \\
\hline PDW (\%) & 15,0 a 17,0 & $11,2 \pm 1,3$ & $11,1 \pm 1,7$ & $t=0,4$ & 0,6000 \\
\hline P-LCR (\%) & 15,8 & $25,7 \pm 5,4$ & $24,8 \pm 7,0$ & $U=3.046,0$ & 0,3000 \\
\hline TP (seg) & 10,0 a 14,0 & $10,3 \pm 3,9$ & $10,6 \pm 1,8$ & $U=2.287,0$ & 0,0007 \\
\hline TAP (\%) & 70,0 a 100,0 & $92,5 \pm 13,0$ & $89,5 \pm 14,2$ & $t=1,3$ & 0,1000 \\
\hline INR & 0,8 a 1,0 & $0,9 \pm 0,1$ & $1,0 \pm 0,2$ & $U=2.300,0$ & 0,0008 \\
\hline TTPA (seg) & 24,0 a 40,0 & $33,8 \pm 8,2$ & $36,9 \pm 8,6$ & $U=2.606,5$ & 0,0100 \\
\hline
\end{tabular}

Fonte: Elaborada pelos autores, 2021.

*Valores de referência conforme Sociedade Brasileira de Análises Clínicas; bulas dos reagentes utilizados; Melo e Silveira ${ }^{60}$ e Henry ${ }^{40}$.

VCM: Volume corpuscular médio; HCM: Hemoglobina corpuscular média; CHCM: Concentração de hemoglobina corpuscular média); RDW: Coeficiente de variação do VCM; VPM: Volume plaquetário médio; PDW: Amplitude de distribuição de plaquetas; P-LCR: Percentual de plaquetas grandes; TP: Tempo de protrombina; TAP: Atividade da protrombina; INR: Razão de normatização internacional; TTPA: Tempo de tromboplastina parcial ativada; MD: Valores médios; DP: Desvio-padrão; U: Teste estatístico Mann-Whitney; t: Teste estatístico t-student. 
grupo exposto e 99 do não exposto, todos do sexo feminino, enquanto no estudo acima participaram cerca de 50 pessoas, de ambos os sexos no grupo exposto e 50 no grupo controle.

Porém, o presente estudo e os de Lima ${ }^{13,14}$ estão em concordância quanto às alterações na morfologia plaquetária, nos quais se encontraram frequentes macroplaquetas e plaquetas gigantes.

A literatura não detalha tanto a correlação entre testes de coagulação e a presença de metais no organismo. Entretanto, uma vez que os fatores de coagulação são produzidos no fígado, considerando que alguns metais se acumulam neste órgão e dependendo da concentração desses elementos ou de sua simples presença, podem comprometer seu funcionamento causando alterações hematológicas alterando os exames laboratoriais.

Como exemplos, têm-se: o As que, após ser absorvido, se acumula primeiramente no fígado, interferindo nas atividades enzimáticas; o Pb que causa alterações no sistema hematológico; o Cu que tem o fígado como responsável por sua homeostase e, quando em excesso, sobrecarrega os hepatócitos degenerando o órgão; e o Fe que, quando em excesso, é armazenado como ferritina ou hemosiderina, principalmente no fígado e no baço7,27,28,42,43,44,45.

Os testes laboratoriais avaliadores dos fatores de coagulação são importantes parâmetros para monitoramento da coagulação sanguínea. Dentre estes, o TP avalia as vias extrínseca e comum da coagulação. É um teste solicitado para realização de exame pré-operatório, na investigação de coagulopatia ou para monitorar tratamento com anticoagulante oral ${ }^{43,46}$.

O INR visa diminuir a variação no resultado de TP entre os diferentes laboratórios clínicos e é muito utilizado para controle da anticoagulação oral, prevenindo fenômenos tromboembólicos e tornando-se um recurso efetivo para reduzir essas complicações ${ }^{40,43,46,47,48,49}$.

O TTPA avalia o funcionamento da via intrínseca e comum da coagulação, detectando deficiências dos fatores VIII, IX, XI e XII, precalicreína e cininogênio de alto peso molecular, dentre outros.

Portanto, é importante que mais estudos sejam realizados para avaliar se as alterações hematológicas de coagulação são provenientes da presença de metal no organismo.

\section{Alterações morfológicas}

Foram analisados esfregaços sanguíneos das participantes de ambos os grupos e observou-se a frequência de ocorrências das principais alterações morfológicas. No grupo exposto, se destacam presença de macroplaquetas em $38,8 \%$ das amostras, seguida de anisocitose $(28,3 \%)$, poiquilocitose $(28,3 \%)$ e hipocromia $(20,8 \%)$, além de $8,0 \%$ de plaquetas gigantes, enquanto o grupo não exposto apresentou 2,0\% de macroplaquetas, $17,0 \%$ de anisocitose, 5,0\% de poiquilocitose, 9,0\% de hipocromia e ausência de plaquetas gigantes.

As plaquetas são fragmentos citoplasmáticos de megacariócitos, anucleados, discoides, produzidos pela medula óssea, responsáveis pela homeostasia primária. As macroplaquetas apresentam tamanho de 4 a $7 \mu \mathrm{m}$ e plaquetas gigantes geralmente 10 a $20 \mu \mathrm{m}$ (Figura 1A). A formação de macroplaquetas e plaquetas gigantes está relacionada à renovação plaquetária acelerada, sendo demonstrada através do aumento do VPM46,50,51,52.

O estudo realizado com a população feminina e masculina do Elesbão, apresentou elevado quantitativo de macroplaquetas, assim como a presença da poiquilocitose com destaque para dacriócitos e equinócitos no grupo exposto ${ }^{13,14}$.

No grupo exposto do presente estudo, observou-se frequente poiquilocitose com presença de estomatócitos, dacriócitos e equinócitos, assim como a presença de hipocromia (Figura 1B). Os estomatócitos, eritrócitos com halo central chamado estoma e semelhante à boca de peixe (Figura $1 \mathrm{C}$ ) ocorrem ocasionalmente em distensões de sangue normal e na estomatocitose hereditária, hepatopatias, alcoolismo e também devido a exposição dos eritrócitos a substâncias catiônicas e medicamentos ${ }^{43,53}$.

Os dacriócitos (Figura 1D) estão presentes em patologias do tipo mielofibrose, anemias hemolíticas adquiridas, anemia megaloblástica, hiperesplenismo. Equinócitos, hemácias com alteração em sua forma discoide, apresentando-se cobertas de 10 a 30 espículas com forma e distribuição relativamente regulares (Figura 1E), podem estar presente em doença renal e hepática, além de ser artefato de preparo da extensão $(\mathrm{pH}$ alcalino do vidro) ou excesso de EDTA ${ }^{43,53}$.

Testes bioquímicos (marcadores da função hepática e renal)

Baseando-se nos resultados dos marcadores hepáticos e renais dos grupos estudados encontramos diferença significativa nos parâmetros: bilirrubina total $(p=0,0001)$, bilirrubina direta $(p=0,0003)$, bilirrubina indireta $(p=0,0081)$, fosfatase alcalina $(p=<0,0001)$ e creatinina ( $p<0,0001)$ (Tabela 4$)$, com concentrações médias dentro dos valores de referência.

Embora as concentrações médias dos grupos estudados estejam dentro dos intervalos de referência preconizados, observa-se que a fosfatase alcalina (FAL) da população exposta é mais elevada do que a da não exposta, com $12,0 \%$ das mulheres expostas apresentando FAL acima do máximo permitido, enquanto apenas $3,0 \%$ das não expostas apresentaram valores alterados.

Considerando que a exposição aos metais pode causar hepatopatias, os dados bioquímicos podem estar correlacionados com os metais detectados nas águas de consumo estudadas. $0 \mathrm{Mn}$, quando em excesso no organismo, encontra-se em níveis mais elevados no fígado, conjugado aos sais biliares. $\mathrm{O}$ excesso de $\mathrm{Fe}$ no sangue provoca a hemocromatose, doença que acumula ferro na forma de ferritina nos músculos, fígado, pâncreas, articulações provocando danos a eles. 0 As acumula-se principalmente no fígado, assim como o $\mathrm{Pb}^{41,42,54}$.

Neste estudo, as transaminases hepáticas (AST, ALT) não apresentaram diferença significativa entre os grupos, não corroborando com outro estudo realizado no qual se observou significância para a ALT na população feminina estudada ${ }^{13,14}$. Entretanto, em ambos, os resultados da população exposta estão maiores do 

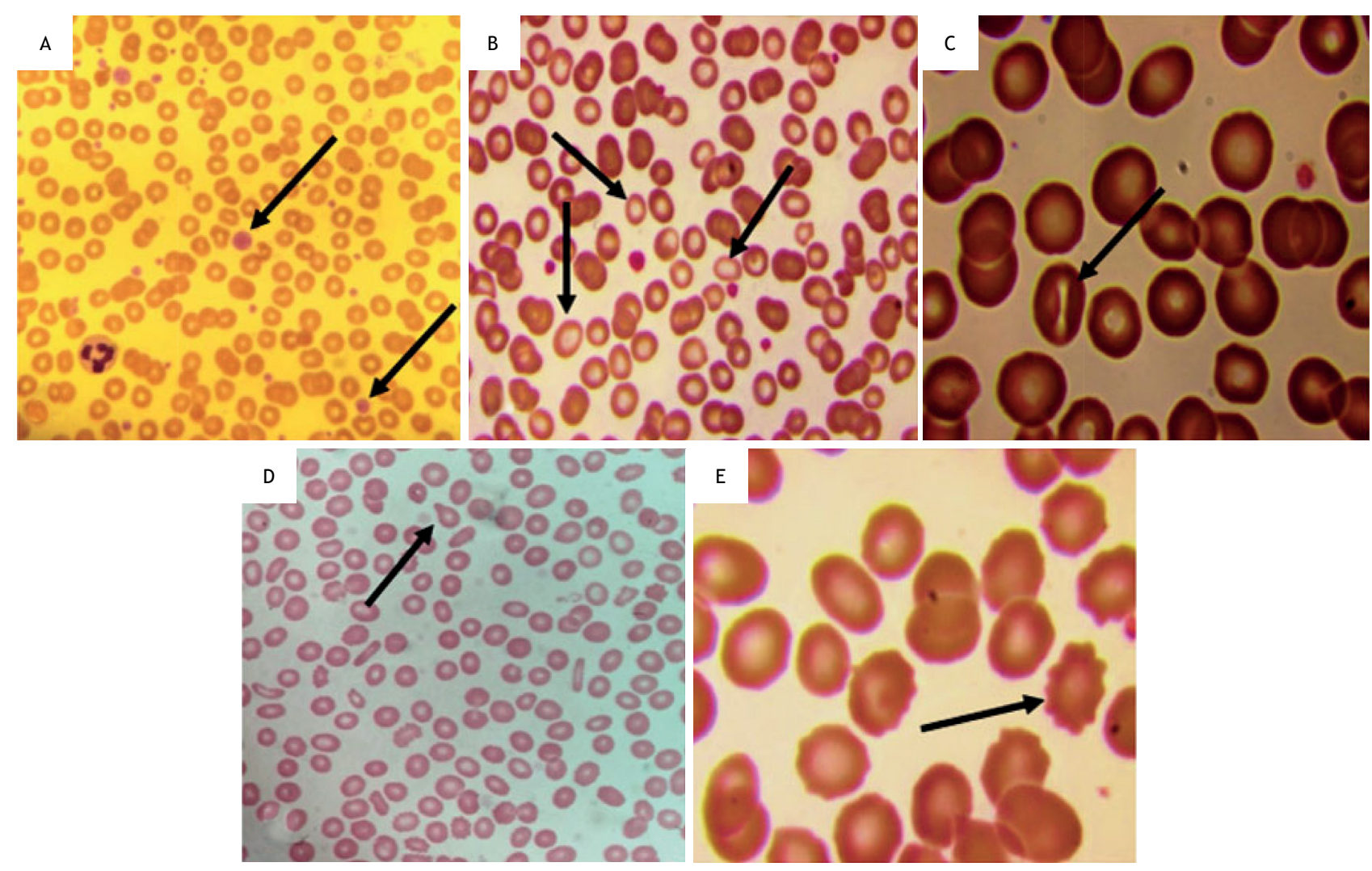

Fonte: Elaborada pelos autores, 2021

Figura 1. (A) Macroplacas e Plaquetas Gigantes, (B) Hipocromia e Anisocitose, (C) Estomatócitos, (D) Dacriócitos, (E), Equinócitos.

Tabela 4. parâmetros bioquímicos bilirrubina total, bilirrubina direta, bilirrubina indireta, fosfatase alcalina (FAL), AST/TGO, ALT/TGP, Gama GT, ureia e cretainina realizados nos grupos exposto e não exposto. Valores médios (MD), desvio-padrão (DP), teste estatístico e valor de p.

\begin{tabular}{|c|c|c|c|c|c|}
\hline \multirow{3}{*}{ Exames bioquímicos } & \multirow{3}{*}{$\mathrm{VR}^{*}$} & \multicolumn{2}{|c|}{ Grupo } & \multirow{3}{*}{ Resultado do teste } & \multirow{3}{*}{ p-valor } \\
\hline & & Exposto & Não exposto & & \\
\hline & & $M D \pm D P$ & $M D \pm D P$ & & \\
\hline Bilirrubina total & Até 1,2 & $0,3 \pm 0,2$ & $0,4 \pm 0,2$ & $t=-3,90$ & 0,0001 \\
\hline Bilirrubina direta & Até 0,4 & $0,1 \pm 0,1$ & $0,1 \pm 0,1$ & $t=-3,70$ & 0,0003 \\
\hline Bilirrubina indireta & Até 0,8 & $0,2 \pm 0,1$ & $0,3 \pm 0,2$ & $U=2.511,50$ & 0,0080 \\
\hline Fosfatase alcalina & 27,0 a 100,0 & $73,3 \pm 25,0$ & $50,0 \pm 24,6$ & $t=5,90$ & $<0,0001$ \\
\hline AST/TGO (U/L) & Até 42,0 & $22,8 \pm 9,1$ & $20,9 \pm 8,4$ & $U=2.935,50$ & 0,2000 \\
\hline ALT/TGP (U/L) & Até 42,0 & $20,0 \pm 10,2$ & $19,5 \pm 12,7$ & $t=-1,80$ & 0,0600 \\
\hline Gama GT(U/L) & 5,0 a 58,0 & $36,7 \pm 28,1$ & $37,5 \pm 29,6$ & $t=-0,09$ & 0,9000 \\
\hline Ureia & 10,0 a 45,0 & $30,3 \pm 9,8$ & $31,7 \pm 8,2$ & $t=-0,70$ & 0,4000 \\
\hline Creatinina & 0,4 a 1,3 & $0,5 \pm 0,2$ & $0,8 \pm 0,2$ & $t=-7,00$ & $<0,0001$ \\
\hline
\end{tabular}

Fonte: Elaborada pelos autores, 2021.

*VR: Valor de referência de acordo com os kits reagentes de bioquímica Labtest utilizados nas dosagens. AST/TGO: Aspartato aminotransferase ou transaminase oxalacética; ALT/TGP: Alanina aminotransferase; U: Teste estatístico Mann-Whitney; t: Teste estatístico t-student.

que a do grupo controle mesmo, encontrando-se dentro do valor de referência preconizado.

A exposição crônica aos metais pode influenciar esses resultados, entretanto, a creatinina pode ser alterada por vários fatores, como o esforço físico, ação de alguns medicamentos e desnutrição ${ }^{55,56}$. Segundo Ramos e Marini ${ }^{57}$, a elevação do nível sérico de creatinina pode ser encontrada na necrose muscular esquelética ou atrofia, insuficiência cardíaca congestiva, choque, diarreia ou fístulas gastrointestinais, diabetes não controlado, dentre outros $^{57}$. Fatores como etnia, raça, idade e sexo também influenciam no nível sérico de creatinina ${ }^{58}$.

Um estudo que realizou o comparativo entre grupos dos marcadores da função renal na população do Elesbão não encontrou significância estatística. No entanto, em sua avaliação renal individual, evidenciou-se que sete indivíduos do grupo exposto apresentaram alterações na creatinina e um na ureia e no não exposto não houve alterações ${ }^{14}$. Ao compararmos a análise dos grupos com o presente estudo, pode ser observado que os resultados da 
ureia corroboraram com o estudo citado acima. Entretanto, os da creatinina apresentaram significância estatística.

\section{CONCLUSÕES}

Após análises em amostras da água do Rio Amazonas e igarapés, utilizada pelas moradoras do Elesbão, foi detectada a contaminação por metais. A exposição prolongada pode comprometer a saúde da população, podendo estar relacionada com as alterações nos exames hematológicos, morfológicos, bioquímicos e de coagulação.

Ao analisar as matrizes biológicas urina e cabelo, encontramos concentrações significativamente elevadas de $\mathrm{Mn}$, principal minério de extração local.
Porém, estudos mais aprofundados e direcionados à saúde da mulher precisam ser realizados com o aumento no número de participantes e a inclusão de outros parâmetros como a análise de hormônios e marcadores genéticos.

A alta presença do metal As na água do rio deve ser avaliada nas matrizes biológicas bem como a inclusão da população de crianças, por se tratar de um grupo especialmente sensível à toxicidade de metais.

Por fim, a investigação de outras fontes de exposição a metais é de extrema importância para que possam ser tomadas medidas de prevenção visando eliminar ou minimizar os riscos dos efeitos adversos relacionados à exposição e, assim, melhorar a qualidade de vida da população.

\section{REFERÊNCIAS}

1. Asmus CIRF, Barbosa AP, Meyer A, Damasceno N, Rosa ACS, Medronho R et al. Rio birth cohort study on environmental exposure and childhood development: pipa project. Ann Global Health. 2020;86(1):1-10. https://doi.org/10.5334/aogh.2709

2. Lima MO, Faial KRF, Brabo ES, Santos ECO, Angélica RS, Mendes RA et al. Avaliação de arsênio total, de elementos traços e bacteriológica em águas de consumo na comunidade do Elesbão, município de Santana, estado do Amapá, Brasil. Cad Saúde Colet. 2007;15(4):467-82.

3. Santos ECO, Jesus IM, Brabo ES, Fayal KF, Sá Filho GC, Lima $M O$ et al. Exposição ao mercúrio e ao arsênio em estados da amazônia: síntese dos estudos do Instituto Evandro Chagas/Funasa. Rev Bras Epidemiol. 2003;6(2):171-85. https://doi.org/10.1590/S1415-790X2003000200010

4. Facundes RS. Danos socioambientais provenientes do manuseio inadequado de rejeitos de manganês e as implicações para a vida e a saúde dos moradores da vila do Elesbão [dissertação]. Macapá: Universidade Federal do Amapá; 2011.

5. Sousa JKC. Avaliação de impactos ambientais causados por metais-traço em água, sedimento e material biológico na Baia de São Marcos, São Luís - Maranhão [doutorado]. João Pessoa: Universidade Federal da Paraíba; 2009.

6. Ramos TD. Avaliação da exposição ambiental ao manganês na população residente no entorno de um estaleiro no município de Angra dos Reis, RJ [dissertação]. Rio de Janeiro: Fundação Oswaldo Cruz; 2013

7. Ruppenthal JE. Toxicologia. Santa Maria: Rede e-Tec Brasil; 2013.

8. Teixeira DCL, Moreira IFV, Coelho MA, Amaral YFQ, Cupertino MC. Exposure to inorganic environmental contaminants and damage to human health. Braz J Health Rev. 2020;3(4):10353-69. https://doi.org/10.34119/bjhrv3n4-256

9. Ministério da Saúde (BR). Política nacional de atenção integral à saúde da mulher: princípios e diretrizes. Brasília: Ministério da Saúde; 2004.

10. Lobo F. Metais tóxicos e suas consequências para a saúde humana. EcoDebate. 1 ago 2011[acesso 10 fev 2020].
Disponivel em: https://www.ecodebate.com.br/2011/08/01/ metais-toxicos-e-suas-consequencias-para-a-saude-human a-artigo-de-frederico-lobo

11. Rodrigues $\mathrm{CV}$. Efeito mutagênico ambiental de metais pesados em mulheres de Santo Amaro da Purificação, Bahia [dissertação]. Salvador: Universidade Federal da Bahia; 2002 [acesso 7 abr 2020]. Disponível em: https://www.arca.fiocruz.br/handle/icict/34260.

12. Liang X, Feswickb A, Simmonsc D, Martyniukd CJ. Environmental toxicology and omics: a question of sex. J Proteomics. 2018;172:152-64. https://doi.org/10.1016/j.jprot.2017.09.010

13. Lima SEA, Almeida AF, Bezerra RM, Braga FS, Azevedo BO, Milhomen WMC et al. Biomonitoring through the laboratory evaluation of a population exposed to ores. Rev Cienc Farm Básica Apl. 2017;38(1):1-9.

14. Lima SEA. Biomonitoramento através da avaliação laboratorial de uma população exposta a metais no município de Santana, Amapá, Brasil [dissertação]. Macapá: Universidade Federal do Amapá; 2018.

15. American Public Health Association - APHA. Standard methods for the examination of water and watwater. 22a ed. Washington: American Public Health Association; 2012.

16. Lima DP. Avaliação da contaminação por metais pesados na água e nos peixes da bacia do rio Cassiporé, estado do Amapá, Amazônia, Brasil [dissertação]. Macapá: Universidade Federal do Amapá; 2013.

17. Lima DP, Santos C, Silva RS, Yoshioka ETO, Bezerra RM. Contaminação por metais pesados em peixes e água da bacia do rio Cassiporé, Estado do Amapá, Brasil. Acta Amaz. 2015;45(4):405-14. https://doi.org/10.1590/1809-4392201403995

18. Conselho Nacional do Meio Ambiente - Conama. Resolução $\mathrm{N}^{\circ} 357$, de 17 de março de 2005. Dispõe sobre a classificação dos corpos de água e diretrizes ambientais para o seu enquadramento, bem como estabelece as condições e padrões de lançamento de efluentes, e dá outras providências. Diário Oficial União. 18 mar 2005. 
19. Ministério da Saúde (BR). Coleta de sangue: diagnóstico e monitoramento das DST, Aids e hepatites virais. Brasília: Ministério da Saúde; 2010.

20. Pozebon D, Dressler VL, Curtius AJ. Análise de cabelo: uma revisão dos procedimentos para a determinação de elementos traço e aplicações. Quim Nova. 1999;22(6):838-46. https://doi.org/10.1590/S0100-40421999000600011

21. Frazão SV. Estudo da determinação de elementos traço em cabelos humanos pelo método de análise por ativação com nêutrons [mestrado]. São Paulo: Universidade de São Paulo; 2008.

22. Moreau RLM, Siqueira MEPB. Ciências farmacêuticas: toxicologia analítica. 2a ed. Rio de Janeiro: Guanabara Koogan; 2016.

23. Mendes SUR. Avaliação dos teores de mercúrio em crianças residentes em área de garimpo no município de Chapada de Natividade, Tocantins [dissertação]. São Paulo: Universidade de São Paulo; 2017.

24. Marinha MSS. Níveis de cádmio em sangue e urina dos habitantes do entorno de uma reserva ecológica no pantanal mato-grossense [dissertação]. Rio de Janeiro: Fundação Oswaldo Cruz; 2011.

25. Noronha KVMS, Andrade MV. 0 efeito da distribuição de renda sobre o estado de saúde individual no Brasil. Rev Pesq Plan Econ. 2008;37(3):521-56.

26. Marinho IG, Lima Junior CAA, Cardoso AKM, Barboza NM, Menezes RAO. Fatores de risco para doenças crônicas não-transmissíveis em moradores no entorno de áreas alagadas na periferia de Macapá, Amapá. Braz J Health Rev. 2018;1(1):148-63.

27. Pinheiro $M O$, Souza $C B$. Efeitos teratogênicos dos metais pesados sobre a infertilidade humana e malformações congênitas. Rev Unilus Ens Pesq. 2017;14(35):47-58.

28. Burtis CA, Burns DE. Fundamentos de química clínica e diagnóstico molecular. Rio de Janeiro: Elsevier; 2012.

29. Alvarenga KF, Morata TC, Lopes AC, Feniman MR, Corteletti LCBJ. Brainstem auditory evoked potentials in children with lead exposure. Braz J Otorhinolaryngol. 2015;81(1):37-43. https://doi.org/10.1016/j.bjorl.2013.12.001

30. Moreira FR, Moreira JC. Os efeitos do chumbo sobre o organismo humano e seu significado para a saúde. Rev Panam Salud Publica. 2004:15(2):119-29.

31. Moreira MFR, Ferreira AP, Araújo UC. Determinação dos níveis de exposição de metais em trabalhadores da construção naval: impactos e desafios. Rev Bras Med Trab. 2016;14(1):19-28.

32. Cunha EL. Avaliação da contaminação bacteriana e por metais pesados na orla fluvial do município de Macapá, Amapá [dissertação]. Macapá: Universidade Federal do Amapá; 2012.

33. Viana GFS. Avaliação da exposição ambiental ao manganês por marcadores não invasivos e efeitos neuropsicológicos em adultos [dissertação]. Salvador: Universidade Federal da Bahia; 2013.

34. Menezes-Filho JA, Paes CR, Pontes AC, Moreira JC, Sarcinelli PN, Mergler D. High levels of hair manganese in children living in the vicinity of a ferro-manganese alloy production plant. Neurotoxicology. 2009;30(6):1207-13. https://doi.org/10.1016/j.neuro.2009.04.005

35. Paulelli ACC. Avaliação da exposição a elementos químicos em moradores residentes em áreas atingidas pelo rompimento da barragem de rejeitos de minério de ferro em Mariana (MG) [doutorado]. São Paulo: Universidade de São Paulo; 2019.

36. Saraiva ACF, Pereira SFP. Avaliação de chumbo em cabelo de populações de área de garimpo: região da volta grande do rio Xingu. In: Anais do $5^{\circ}$ Congresso Brasileiro de Pesquisas Ambientais e Saúde; Santos, Brasil. Santos: Council of Researches in Education and Sciences; 2005.

37. Williamson MAL, Snyder M. Interpretação de exames laboratoriais. 9a ed. Rio de Janeiro: Guanabara Koogan; 2013.

38. Sá PGS. Contaminação do ambiente marinho por metais pesados e suas implicações sobre comunidades de pescadores artesanais [doutorado]. Rio de Janeiro: Fundação Oswaldo Cruz; 1999.

39. Failace R. Hemograma: manual de interpretação. Porto Alegre: Artmed; 2009.

40. Henry JB. Diagnósticos clínicos e tratamento por métodos laboratoriais. 21a ed. São Paulo: Manole; 2013.

41. Xu J, Zhao M, Pei L, Liu X, Wei L, Li A et al. Effects of heavy metal mixture exposure on hematological and biomedical parameters mediated by oxidative stress. Sci Total Environ. 2020;705. https://doi.org/10.1016/j.scitotenv.2019.134865

42. Baierle M, Valentine J, Paniz C, Moro A, Barbosa JR F, Garcia SC. Possíveis efeitos do cobre sanguíneo sobre parâmetros hematológicos em idosas. J Bras Patol Med Labor. 2010;46(6):463-70. https://doi.org/10.1590/S1676-24442010000600006

43. Silva PH, Alves HB, Comar SR, Hennemberg R, Merlin JC, Stinguen ST. Hematologia laboratorial: teoria e procedimentos. Porto Alegre: Artmed; 2016.

44. Squitti R, Guidoni R, Simonelli I, Ivanova ID, Colabufo NA, Zuin $M$ et al. Copper dyshomeostasis in Wilson disease and Alzheimer's disease as shown by serum and urine copper indicators. J Trace Elem Med Biol. 2018;45:181-8. https://doi.org/10.1016/j.jtemb.2017.11.005

45. Hu Y, Zhou J, Du B, Liu H, Zhang W, Liang J et al. Health risks to local residents from the exposure of heavy metals around the largest copper smelter in China. Ecotoxicol Environ Saf. 2019;171:329-36. https://doi.org/10.1016/j.ecoenv.2018.12.073

46. Naoum FA. Doenças que alteram os exames hematológicos. Rio de Janeiro: Atheneu; 2010.

47. Hospital Albert Einstein. Diretrizes assistenciais: protocolo de anticoagulação oral. São Paulo: Hospital Albert Einstein; 2012[acesso 21 nov 2019]. Disponível em: https:// docplayer.com.br/21403022-Diretrizes-assistenciais.html

48. Araújo ACO, Domingues RB, Bellen BV. Determinação do INR: comparação entre método convencional e dispositivo portátil. J Vasc Bras. 2014;13(2):88-93. https://doi.org/10.1590/jvb.2014.051 
49. Camargos AMT, Giarola FC, Rocha F, Castro IA, Fonseca LHR, Cardoso Júnior MD et al. Protocolo clínico: monitorização de pacientes em uso de varfarina ambulatório universitário. Divinópolis: Universidade Federal de São João Del Rei; 2017.

50. Castro HC, Ferreira BLA, Nagashima T, Schueler A, Rueff C, Camisasca D et al. Plaquetas: ainda um alvo terapêutico. J Bras Patol Med Lab. 2006;42(5):321-32. https://doi.org/10.1590/S1676-24442006000500004

51. Farias MG, Bó SD. Importância clínica e laboratorial do volume plaquetário médio. J Bras Patol Med Lab. 2010;46(4):275-81. https: / /doi.org/10.1590/S1676-24442010000400003

52. Pereira AV, Rocha FDLM, Oliveira AN, Tapety FI, Cavalcante AACM, Chaves TVSC. Haematological and genotoxic profile study of worker exposed to medical waste. Rev Pesq Cuid Fundam. 2013;5(6):160-8. https: / / doi.org/10.9789/2175-5361.rpcfo.v5.3397

53. Bain BJ. Células sanguíneas: um guia prático. Porto Alegre: Artmed; 2016.

54. US Department of Health and Human Services - DHHS. Toxicological profile for manganese. Atlanta: Department of Health and Human Services; 2012[acesso 4. ago 2020]. Disponível em: http://www.atsdr.cdc.gov/toxprofiles/tp151.pdf
55. Dalton RN. Creatinina sérica e taxa de filtração glomerular: percepção e realidade. J Bras Patol Med Lab. 2011;47(1):8-11. https://doi.org/10.1590/S1676-24442011000100001

56. Morales AP, Maciel RN, Jorge FS, Arêas Neto NT, Cordeiro DC, Viana MAS et al. Alterações dos níveis séricos de creatinina, ácido úrico, creatina kinase e da taxa de filtração glomerular em corredores de "rua". Rev Bras Cineantropom Desempenho Hum. 2013;15(1):71-81. https://doi.org/10.1590/1980-0037.2013v15n1p71

57. Ramos G, Marini DC. Exames bioquímicos relacionados a alterações renais. Foco Cad Est Pesq. 2014;5(6):11-26.

58. Brito TNS, Oliveira ARA, Silva AKC. Taxa de filtração glomerular em adultos: características e limitações das equações utilizadas. Rev Bras Anal Clín. 2016;48(1):7-12.

59. Ministério do Trabalho (BR). Norma regulamentadora $N^{\circ} 7$, de 5 de julho de 1978. Estabelece a obrigatoriedade de elaboração e implementação, por parte de todos os empregadores e instituições que admitam trabalhadores como empregados, do programa de controle médico de saúde ocupacional PCMSO, com o objetivo de promoção e preservação da saúde do conjunto dos seus trabalhadores. Diário Oficial União. 6 jul 1978.

60. Melo MAW, Silveira CM. Laboratório de hematologia: teorias, técnicas e atlas. Rio de Janeiro: Rubio; 2015.

Contribuição dos Autores

Cardoso FMN, Gomes MRF - Concepção, planejamento (desenho do estudo), aquisição, análise, interpretação dos dados e redação do trabalho. Cardoso FMN, Telles CR, Milhomem WMC, Trindade Junior SV, Vidal LJG, Bezerra RM, Braga FS, Vasconcelos HCG, Almeida AF, Lima SEA - Aquisição, análise e interpretação dos dados. Todos os autores aprovaram a versão final do trabalho.

Conflito de Interesse

Os autores informam não haver qualquer potencial conflito de interesse com pares e instituições, políticos ou financeiros deste estudo.

Licença CC BY-NC atribuição não comercial. Com essa licença é permitido acessar, baixar (download), copiar, imprimir, compartilhar, reutilizar e distribuir os artigos, desde que para uso não comercial e com a citação da fonte, conferindo os devidos créditos de autoria e menção à Visa em Debate. Nesses casos, nenhuma permissão é necessária por parte dos autores ou dos editores. 